$I-33032$ DR. $0363-8$

ANL/MCS-TM-100

cat.

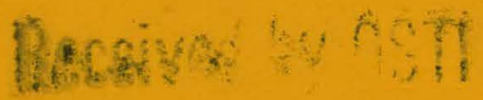

JAN 191988

\title{
AN ADAPTIVE PSEUDO-SPECTRAL METHOD FOR REACTION DIFFUSION PROBLEMS
}

by

A. Bayliss, D. Gottlieb, B. J. Matkowsky, M. Minkoff

DO NOT MICROFILM COVER

October 1987

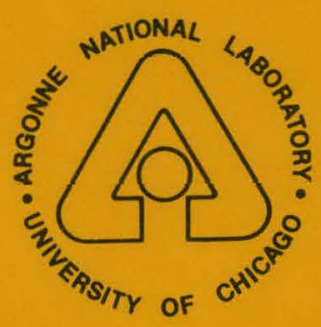

MATHEMATICS AND COMPUTER SCIENCE DIVISION 


\section{DISCLAIMER}

This report was prepared as an account of work sponsored by an agency of the United States Government. Neither the United States Government nor any agency Thereof, nor any of their employees, makes any warranty, express or implied, or assumes any legal liability or responsibility for the accuracy, completeness, or usefulness of any information, apparatus, product, or process disclosed, or represents that its use would not infringe privately owned rights. Reference herein to any specific commercial product, process, or service by trade name, trademark, manufacturer, or otherwise does not necessarily constitute or imply its endorsement, recommendation, or favoring by the United States Government or any agency thereof. The views and opinions of authors expressed herein do not necessarily state or reflect those of the United States Government or any agency thereof. 


\section{DISCLAIMER}

Portions of this document may be illegible in electronic image products. Images are produced from the best available original document. 


\section{The following pages are an exact representation of what is in the original document folder.}


Argonne National Laboratory, with facilities in the states of Illinois and Idaho, is owned by the United States government, and operated by The University of Chicago under the provisions of a contract with the Department of Energy.

\section{DISCLAIMER}

This report was prepared as an account of work sponsored by an agency of the United States Government. Neither the United States Government nor any agency thereof, nor any of their employees, makes any warranty, express or implied, or assumes any legal liability or responsibility for the accuracy, completeness, or usefulness of any information, apparatus, product, or process disclosed, or represents that its use would not infringe privately owned rights. Reference herein to any specific commercial product, process, or service by trade name, trademark, manufacturer, or otherwise, does not necessarily constitute or imply its endorsement, recommendation, or favoring by the United States Government or any agency thereof. The views and opinions of authors expressed herein do not necessarily state or reflect those of the United States Government or any agency thereof. 


\title{
AN ADAPTIVB PSEUDO-SPECTRAL MBTHOD \\ FOR REACTION DIFFUSION PROBLETS
}

\author{
by \\ A. Bayliss $1,2,3$ \\ Department of Engineering Sciences and Applied Mathematics \\ Northwestern Iniversity \\ Evanston, IL 60208 \\ D. Gottlieb 3,5 \\ Division of Applied Mathematics, Box F \\ - Brown University \\ Providence, RI 02912 \\ B. J. Matkowsky ${ }^{1,2}$ \\ Department of Engineerings Sciences and Applied Mathematics \\ Northwestern University \\ Evanston, IL 60208 \\ M. Minkof $f^{1}$ \\ Mathematics and Computer Science Division \\ Argonne National Laboratory \\ Argonne, IL 60439 \\ Mathematics and Computer Science Division \\ Technical Memorandum No. 100
}

October 1987

${ }^{1}$ Supported by the Applied Mathematical Sciences subprogram of the Office of Energy Research, U.S. Department of Energy under Contract W-31-109-ENG-38.

${ }^{2}$ Supported by the Applied Mathematical Sciences subprogram of the Office of Energy Research, U.S. Department of Fnergy under Grant DFFG02-87ER-25027.

${ }^{3}$ Supported by the National Aeronautics and Space Administration under NASA Contract No. NAS1-18107 while in residence at the Institute for Computer Applications in Science and Engineering (ICASE), NASA Langley Research Center, Hampton, VA 23665.

${ }^{4}$ Supported by NSF Grant DMS-8701543.

5 Research was supported by AFOSR Grant AF-85-0303.

The computations in this technical memorandum were conducted with support from the Scientific Computing Staff of the Applied Mathematical Sciences subprogram of the Office of Energy Research, U.S. Department of Energy. 
THIS PAGE

\section{WAS INTENTIONALLY LEFT BLANK}




\section{Table of Contents}

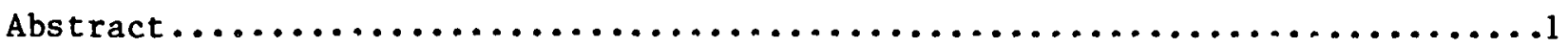

I. Introduction. .......................................

II. Problem Description....................................

III. Adaptive Procedure......................................4

IV. The New Functiona1...................................

V. Numerical Results...................................9

VI. Conclusion.........................................11

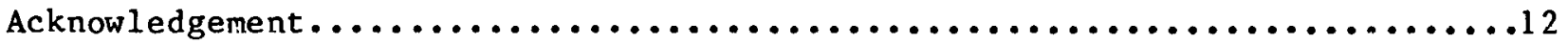

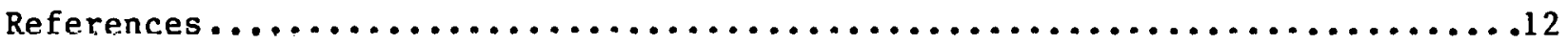




\section{List of Figures}

1. $\theta$ and $\mathrm{C}$ as a function of time at a fixed radial location...........13

2a. Spatial profiles of $\theta$ at four different times.................14

2b. Spatial profiles of $\mathrm{C}$ at four different times...................

3a. $\theta, C$, and $J$ as a function of time. $J$ and $\underset{\sim}{\alpha}$ were

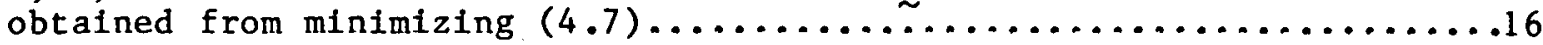

3b. $\theta, C$, and $J$ as a function of time. $J$ and $\underset{\sim}{\alpha}$ were obtained from minimizing $(3.5) \ldots \ldots \ldots \ldots \ldots \ldots \ldots \ldots \ldots \ldots \ldots \ldots$

4a. Spatial profiles of $\odot$ and $C$ at the time when the objectivo functional is largest. J and a were obtained from minimizing $(4.7)$ and $\varepsilon=.0025 \ldots \ldots \ldots \ldots \ldots \ldots \ldots \ldots 18$

4b. Spatial profiles of $\theta$ and $C$ at the time when the objective functional is largest. $J$ and $\alpha$ were obtained from minimizing $(3.5)$ and $\varepsilon=.0025 \ldots \ldots \ldots \ldots \ldots \ldots \ldots \ldots$

5a. Spatial profiles of $\theta$ and $C$ at the time when the objective functional is largest. $J$ and $\alpha$ were

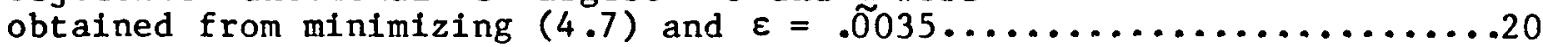

5b. Spatial profiles of $\theta$ and $C$ at the time when the objective functional is largest. $J$ and $\alpha$ were

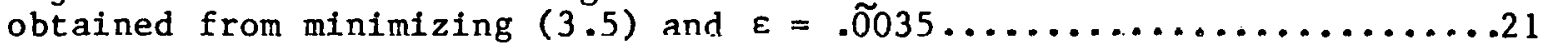

6a. Spatial profiles of $\theta$ and $C$ at the time when the objective functional is largest. $J$ and $\alpha$ were obtained from minimizing $(4.7)$, with $\mathrm{J}$ constrained to be $\leq 241 \ldots \ldots \ldots \ldots \ldots \ldots 22$

6b. Spatial profiles of 0 and $C$ at the time when the objective functional is largest. $J$ and $\alpha$ were obtained from minimizing (3.5), with J constraine $\tilde{\text { d }}$ to be $\leq 241 \ldots \ldots \ldots \ldots \ldots$

7a. Angular variation of $\Theta$ and $C$ for a cellular flame; $r=9.34 \ldots \ldots \ldots . .24$

7b. Angular variation of $\Theta$ and $C$ for a cellular flame; $r=10.15 \ldots \ldots \ldots$

7c. Mean, fundamental and harmonic for a cellular flame,

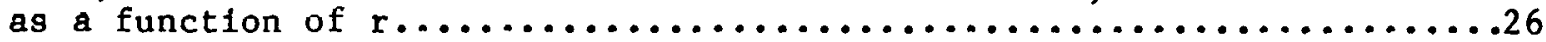

8a. Mean of $\odot$ as a function of $r$. Coarse grid solution obtained from minimizing $(4.7) \ldots \ldots \ldots \ldots \ldots \ldots \ldots \ldots \ldots \ldots \ldots \ldots \ldots \ldots$

8b. Fundamental and harmonic of $\theta$ as a function of $r$. Coarse grid solution obtained from minimizing $(4.7) \ldots \ldots \ldots \ldots \ldots \ldots \ldots 29$

9a. Mean of $\odot$ as a function of $r$. Coarse grid solution

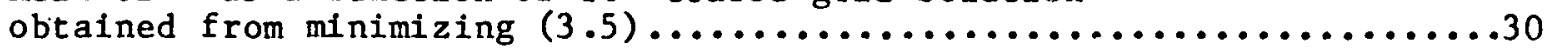

$9 \mathrm{~b}$. Fundamental and harmonic of $\theta$ as a function of $r$. Coarse grid solution obtained from minimizing $(3.5) \ldots \ldots \ldots \ldots \ldots \ldots \ldots 1$ 


\title{
AN ADAPTIVE PSEUDO-S PECT RAL METHOD FOR REACTION DIFFUSION PROBLEMS
}

\author{
by \\ A. Bayliss, D. Gottlieb, B. J. Matkowsky, M. Minkoff
}

\begin{abstract}
We consider the spectral interpolation error for both Chebyshev pseudo-spectral and Galerkin approximations. We develop a family of functionals $I_{r}(u)$, with the property that the maximum norm of the error is bounded by $I_{r}(u) / J^{r}$, where $r$ is an integer and $J$ is the degree of the polynomial approximation. These functionals are used in an adaptive procedure whereby the problem is dynamically transformed to minimize $I_{r}(u)$. The number of collocation points is then chosen to maintain a prescribed error bound. The method is illustrated by various examples from combustion problems in one and two dimensions.
\end{abstract}

\section{Introduction}

The objective of this paper is to describe an adaptive pseudo-spectral method for solving systems of reaction, diffusion, convection equations. We introduce a family of functionals which appear to accurately estimate the spectral interpolation error. These functionals are used in an adaptive procedure in which the equation is transformed into a coordinate system in which the functional is minimized. The number of collocation points is dynamically modified to maintain a constant error bound.

In [1], an adaptive procedure was introduced in which a coordinate transformation was chosen to minimize the weighted second Sobolev norm of the solution. A similar adaptive procedure was presented in [6]. Our numerical computations indicate that the new functionals introduced here provide a considerably sharper error estimate and thereby permit a more efficient solution of the differential equations.

The numerical procedure is illustrated with examples of unsteady axisymmetric as well as steady non-axisymmetric flames occurring in gaseous combustion. This problem is described by the diffusional thermal model in which the thermal expansion of the gas is neglected [9]. We further simplify by assuming that the reaction is controlled by a single deficient component so that only one species needs to be accounted for, and assume global one step Arrhenius kinetics. Thus the model consists of a system of reaction, diffusion, convection equations for the temperature and the concentration of the deficient component which limits the reaction. 
Because of the reaction term, the system has an exponential nonlincarity. Spurious numerical oscillations can seriously degrade the accuracy of the computed solution, primarily through the exponentially nonlinear reaction term. Generally, the reaction term is important only in a narrow region, the reaction zone, in which the solution exhibits rapid changes. The Chebyshev pseudo-spectral method is subject to oscillations in regions of large gradients [5]. We have found that the adaptive procedure described here can be effective in reducing these oscillations. An adaptive procedure for a finite difference solution of problems of this type is given in [11].

This paper is organized as follows. In Section 2, we describe a specific model and illustrate the features of the solution which make its numerical computation difficult. In section 3, we descrihe the adaptive procedurc, and in Section 4 we describe the new functionals which are used to estimate the error. In Section 5, we illustrate the effectiveness of the procedure with examples from the model problem described in Section 2 .

\section{Problem Description}

To illustrate the behavior of the solution in typical combustion problems, we study the following problem for a flame stabilized by a line source of fuel. We consider the nondimensional reaction, diffusion, convection system for the reduced temperature $\theta=\frac{T-T}{T_{b}-T_{u}}$ and reactant concentration $C$, where $T_{u}$ and $T_{b}$ represent the temperature in the unburned fresh mixture at the source and the burned temperature at infinity, respectively. In polar coordinates $r$ and $\phi$, the nondimensional equations are

$$
\begin{aligned}
& \theta_{t}=\Delta \theta-\frac{K \theta_{r}}{r}+\frac{C N^{2}(1-\sigma)^{2}}{2 L} \exp (N(1-\sigma)(\theta-1) /(\sigma+(1-\sigma) \theta)) \\
& C_{t}=\frac{\Delta C}{L}-\frac{K C r}{r}-\frac{C N^{2}(1-\sigma)^{2}}{2 L} \exp (N(1-\sigma)(\theta-1) /(\sigma+(1-\sigma) 0)) .
\end{aligned}
$$

Here $\sigma=\mathrm{T}_{\mathrm{u}} / \mathrm{T}_{\mathrm{b}}, \quad \Delta$ is the Laplacian, $\mathrm{N}$ is the non-dimensional activation energy, and the terms on the right side of (2.1), respectively represent diffusion, convection, and a global one-step Arrhenius reaction. The boundary conditions are given by

$$
\Theta=0, \quad c=1 \text { for } r=0 ; \quad \theta+1, \quad c+0 \text { for } r+\infty
$$

The parameters in (2.1) are the Lewis number, $L$, the strength $k$ of the source of fuel, $N$, and $\sigma:$ To limit the size of the computational domain, we impose the boundary conditions at fixed points $r_{1}, r_{2}$, rather than at 0 and $\infty$. We have verified that the solutions of the problems presented here are insensitive to the positions of the artificial boundaries. 
The system (2.1)-(2.2) was analyzed in [8]. It was shown that in the limit $\quad M=N(1-0)+\infty$ there exists an axisymetric solution of the form

$$
\begin{gathered}
\Theta=\left(\frac{r}{K}\right)^{K}+O\left(\frac{1}{M}\right) \\
C=(1-\theta)+O\left(\frac{1}{M}\right) .
\end{gathered}
$$

Furthermore, there exists a critical value of $L, L_{c_{1}}<1$, such that for $\mathrm{L}<\mathrm{L}_{\mathrm{C}_{1}}$ the solution (2.3) is unstable to angular perturbations, and arbitrary initial data close to (2.3), evolve into stationary cells. Computations of such cells are presented in $[2,3]$.

Another regime of diffusional thermal instabilities occurs for values of $\mathrm{L}>1$. Based on the analysis by M. Garbey, et al.*, there exists another critical value of $\mathrm{L}, \mathrm{L}_{\mathrm{c}_{2}}>1$, such that for $\mathrm{L}>\mathrm{L}_{\mathrm{c}_{2}}$ the solution (2.3) loses its stability to a time oscillatory solution via a Hopf bifurcation. We have found this axisymmetric Hopf bifurcation for (2.3) numerically. Increasing $t$ along this bifurcation branch, we find that the sinusoidal pulsations which occur for $L$ near $L_{c_{2}}$ become progressively sharper and steeper and take on the character of relaxation oscillations. At another critical value $\mathrm{L}_{\mathrm{c}_{3}}$, a period doubling secondary bifurcation is identified.

The adaptive procedure developed here will be illustrated by a typical case after the period doubling secondary bifurcation. Specifically, we consider the case $L=13, \sigma=.5, \mathrm{~N}=40$, and $K=11.7$. More physically realistic values of $\mathrm{L}$ occur for other values of the parameter $\sigma, N$, and $K$. In Figure 1, we illustrate the temporal behavior of $\theta$ and $C$ at the fixed point $r=27.4$ over a time interval slightly greater than one period. The sharp spikes of alternating height are apparent. Computing the solution during the spikes consumes most of the cost of the computation.

To see how the spikes relate to the spatial structure of the solution, we exhibit in Figures $2 a$ and $2 b$ the spatial profiles of $\theta$ and $c$ at the four times indicated in Figure 1. It is apparent that for all of the time points, the solution exhibits very rapid changes over a small spatial interval. Note that the concentration profile has a more rapid spatial variation than the 'temperature profile, since $L>1$. It is apparent from Figure $2 \mathrm{a}$ and $2 \mathrm{~b}$ that the solution becomes much sharper at the time when the temporal. spikes occur. In the limit $M+\infty$, both $O$ and $C$ have discontinuous first derivatives. The point of discontinuity is called the flame front, and the reaction term becomes a surface delta function on the front, the strength of which was derived in [9]. The analysis in [8] is based on this limit. For M large but finite, the reaction term is important only in a thin region called the reaction zone.

\footnotetext{
*M. Garbey, G. K. Leaf, H. G. Kaper, and B. J. Matkowsky, unpublished information.
} 
In computing the solution with the Chebyshev pseudo-spectral method, inaccurate resolution of the region of rapid variation can lead to spurious oscillations $[5,12]$. These oscillations can affect the reaction term and give rise to a variety of errors, including computed solutions which appear to be nonperiodic or chaotic. If the oscillations become large enough, negative concentrations occur, which lead to numerical instability.

To efficiently compute solutions using the Chebyshev pseudo-spectral method, we adaptively transform the coordinate system so that in the new coordinate system the solution appears to vary more gradually. In this way the solution can be approximated with a smaller number of collocation points. In addition, it is desirable to dynamically add and subtract collocation points, as the resolution requirements are more severe when the solution spikes. Our procedure is to choose the number of collocation points to maintain a constant error bound.

These constraints give rise to the need for a good definition and estimate of the errors associated with the pseudo-spectral method. In the next two sections, we describe the adaptive method in general and then discuss the functionals we have developed to monitor the spectral interpolation error. Although this analysis is only for the error in approximating a function as a sum of Chebyshev polynomials, the numerical examples indicate that the procedure appears to control the total pseudo-spectral error in solving the equations of the mathematical model.

\section{Adaptive Procedure}

We consider, for purposes of discussion, the model reaction, diffusion, convection equation in one space dimension

$$
u_{t}=u_{x x}+u_{x}+R(u), \cdot-1 \leq x \leq 1
$$

where $R(u)$, is a nonlinear function. The initial and boundary conditions are not important for the purpose of this discussion. In the Chebyshev pseudospectral method, the solution to (3.1) is approximated as a finite sum of Chebyshev polynomials

$$
u=\sum_{j=0}^{J} a_{j}(t) T_{j}(x),
$$

where $T_{j}(x)=\cos \left(j \cos ^{-1} x\right)$ and the coefficients $a_{j}$ are obtained from collocating the solution at the points

$$
\left\{x_{j}=\cos \left(\frac{\pi j}{J}\right), \quad j=0, \cdots, J\right\}
$$


For a discussion of this method, see [5]. The method differs from finite differences in that it is spectrally accurate; i.e., for sufficlently smooth solutions the error decays faster than $J^{-r}$ for any power of $r$. In particular for analytic functions, the error decays exponentially [13]. A precise formulation of this property is discussed below.

It is known that this method can exhibit oscillations when solutions with rapid spatial changes, such as those exhibited in Figures $2 a, b$, are computed. In order to improve the ability of the method to accurately compute such solutions, an adaptive procedure was introduced in [1].

In this procedure, we dynamically vary the coordinate system for ( 3.1$)$. We transform $(3.1)$ by the mapping

$$
x=q(s, \underset{\sim}{)},
$$

where $s$ is the new independent variable. Here $q$ is a given function, and $\approx$ is an unknown parameter vector to be determined. In [1] and in the computations presented below, the function $q$ was taken to be

$$
q(s, \alpha)=\frac{4}{\pi} \tan ^{-1}\left[\alpha_{1} \tan \frac{\pi}{4}\left(s^{\prime}-1\right)\right]+1 \text {, }
$$

where

$$
s^{\prime}=\frac{\alpha_{2}-s}{\alpha_{2} s-1} ; \quad \alpha_{1}>0,-1<\alpha_{2}<1
$$

and $\quad \underset{\sim}{\alpha}=\left(\alpha_{1}, \alpha_{2}\right)$.

To determine $\underset{\sim}{\alpha}$, we suppose that $I_{r}(u)$ is a functional of the solution and that some measure of the error is bounded by $I_{r}(u) / J^{r}$ for some integer $r$. Then $I_{r}(u(q(\bullet, \alpha)))$ is a measure of the error in the coordinate system determined by $\mathscr{\alpha}$. We can then minimize the upper bound on the error by choosing $\underset{\sim}{\alpha}$ such that $\mathrm{I}_{r}(u(q, \alpha)) \equiv \tilde{\mathrm{I}}_{r}(\underset{\sim}{\alpha})$ is minimized. If $\varepsilon$ is a desired error level, then $\mathrm{J}$ can be determined as the smallest integer such that

$$
\mathrm{J}^{\mathrm{r}} \geq \frac{\tilde{\mathrm{I}}_{\mathrm{r}}(\underline{\alpha})}{\varepsilon}
$$

In this procedure, it is necessary to interpolate the solution to the new collocation points $x_{j}=q\left(s_{j}, \alpha\right)$, where $\left\{s_{j}\right\}$ are the Chebyshev collocation points (3.3). This can be accomplished without loss of spectral accuracy by evaluating the global approximation (3.2). From this discussion it is clear that the role of $I_{r}(u)$ is crucial. In $[1,6], I_{r}(u)$ was chosen as the weighted second Sobolev norm

$$
I_{2}(u)=\left[\int_{-1}^{1} \mathrm{~d} x w(x)\left[\left|u_{x x}\right|^{2}+\left|u_{x}\right|^{2}+|u|^{2}\right]\right]^{1 / 2},
$$


where $w(x)$ is the Chebyshev weight function

$$
w(x)=\left(1-x^{2}\right)^{-1 / 2}
$$

The adaptive procedure is therefore to find $\underset{\sim}{\alpha}$ such that

$$
\tilde{I}(\underset{\sim}{)})=\int_{-1}^{1} \mathrm{ds}\left[\left|\mathrm{u}_{s s}\right|^{2}+\left|\mathrm{u}_{s}\right|^{2}+|\mathrm{u}|^{2}\right]_{\mathrm{w}(\mathrm{s})}^{1 / 2}
$$

is minimized.

The choice of (3.5) is motivated by the estimates obtained in [4], where it is shown that if $u$ is smooth, and $p_{J} u$ the Chebyshev interpolant, then the eirui ${ }_{\mathrm{J}}=\mathrm{u}=\mathrm{P}_{\mathrm{J}} \mathrm{u}$ sarisfies

$$
\left\|e_{J}\right\|_{0} \leq c_{r} \frac{\|u\|_{r}}{J}
$$

for $\mathrm{J}>0$. Here \|\|$_{0}$ is the weighted $\mathrm{L}_{2} \cdot$ norm,

$$
\left\|e_{J}\right\|_{0}^{2}=\int_{-1}^{1} d s w(s)\left|e_{J}\right|^{2},
$$

and $" r$ is the weighted $r^{\text {th }}$ Sobolev norm. We make the following remarks about $(3.7)$

a) As is typical in pseudo-spectral methods, (3.7) is a family of error bounds and the error seen in practice is the smallest of the right hand sides of (3.7). Thus, any adaptive procedure can only minimize an upper bound on the error. For the combustion problems discussed here, we choose $r=2$, i.e. (3.5), although when the spatial profiles become more smoothly varying, this may overestimate $\mathrm{J}$.

b) The error bound on the left hand side of (3.7), which is to be bounded, depends on the particular coordinate system that is chosen and may not necessarily relate to the quality of the computed solution.

c) The estimates leading to (3.7) are not sharp:

d) It has been observed that (3.7) does not yield good coordinate systems for $r=1[1,6]$.

e) In practice we find that (3.5) is not sufficiently sensitive to variations in the behavior of the solution, when compared with the functional described below. The use of (3.5) tends to overestimate the number of collocation points J. A discussion of a new family of functionals, which appear to better track the spectral interpolation error, now follows. 


\section{The New Functional}

To better understand the behavior of the error in the Chebyshev method, and to get a better error functional, we start by considering a smooth function

$$
f(x)=\sum_{k=0}^{\infty} a_{k} T_{k}(x) ; \quad a_{k}=\frac{1}{c_{k}} \int_{-1}^{1} \frac{f(x) T_{k}(x)}{\sqrt{1-x^{2}}} d x,
$$

where $c_{0}=2 ; c_{k}=1$ for $k>0$, and its Chebyshev-Galerkin approximation

$$
\mathrm{f}_{\mathrm{J}-1}(\mathrm{x})=\mathrm{P}_{\mathrm{J}-1} \mathrm{f}(\mathrm{x})=\sum_{\mathrm{k}=0}^{\mathrm{J}-1} \mathrm{a}_{\mathrm{k}} \mathrm{T}_{\mathrm{k}}(\mathrm{x})
$$

We are interested in an error estimate that will not depend on the coordinate system; otherwise it will be impossible to know whether the new coordinate s is really better than the original one. The most natural norm in which to measure the error is the maximum norm. Thus we look at the pointwise error

$$
\left|f(x)-f_{J-1}(x)\right|=\left|\sum_{k=J}^{\infty} a_{k} T_{k}(x)\right| \text {. }
$$

Consider first the leading term, $a_{J}$, in (4.1). In fact, since for $k>0$,

$$
\left(\sqrt{1-x^{2}} T_{k}^{\prime}\right)^{\prime}=-\frac{k^{2} T_{k}}{\sqrt{1-x^{2}}} \text {, }
$$

we obtain

$$
a_{J}=-\frac{1}{c_{J} J^{2}} \int_{-1}^{1}\left(\sqrt{1-x^{2}} T_{J}^{\prime}\right)^{\prime} f(x) d x
$$

We then integrate by parts, noting that the boundary terms vanish, to obtain

$$
a_{J}=\frac{1}{c_{j} J^{2}} \int \sqrt{1-x^{2}} T_{J}^{\prime} f^{\prime}(x) d x=-\frac{1}{c_{J} J^{2}} \int_{-1}^{1}\left(\sqrt{1-x^{2}} f^{\prime}\right)^{\prime} T_{J} d x,
$$

or upon defining $L=\sqrt{1-x^{2}} \frac{d}{d x}$,

$$
a_{J}=-\frac{1}{c_{j} J^{2}} \int\left(L^{2} f\right) \frac{T_{J}}{\sqrt{1-x^{2}}} d x
$$

This formula is a special case of the formula derived in [5] for a general Sturm-Liouville problem. We repeat the integration by parts to obtain

$$
a_{J}=\frac{(-1)^{k}}{c_{J} J^{2 k}} \int\left(L^{2 k} f\right) \frac{T_{J}}{\sqrt{1-x^{2}}} d x, \quad k=1,2, \cdots
$$


From (4.4), we can bound $a_{J}$ by using the Cauchy-Schwartz inequali.ty

$$
\left|\mathrm{a}_{J}\right| \leq \frac{1}{\mathrm{~J}^{2}}\left[\int \frac{\left(\mathrm{L}^{2} \mathrm{f}\right)^{2}}{\sqrt{1-\mathrm{x}^{2}}} \mathrm{dx}\right]^{1 / 2} \cdot \frac{\mathrm{l}}{\mathrm{c}_{\mathrm{J}}}\left[\int \frac{\mathrm{T}_{\mathrm{J}}^{2}}{\sqrt{1-\mathrm{x}^{2}}} \mathrm{dx}\right]^{1 / 2} \text {. }
$$

The last factor on the right hand side of (4.6) is one, by the normalization of $\mathrm{T}_{\mathrm{j}}$. It is therefore reasonable to take

$$
I_{2}[f]=\left\{\int_{-1}^{1} \frac{\left(L^{2} f\right)^{2}}{\sqrt{1-x^{2}}} d x\right\}^{\frac{1}{2}}=\left\{\int_{-1}^{1} \frac{d x}{\sqrt{1-x^{2}}}\left[\left(\sqrt{1-x^{2}} \frac{d}{d x}\right)^{2} f\right]^{2}\right\}^{\frac{1}{2}}
$$

instead of (3.5). Similarly, we can define

$$
I_{2 k}[f]=\left[\int_{-1}^{1} \frac{\left(L^{2 k} f\right)^{2}}{\sqrt{1-x^{2}}} d x\right]^{1 / 2}
$$

and take the minimum over $k$ as a measure for the error. Going back to (4.1), we can bound the (Galerkin) spectral interpolation error in the maximum norm

$$
\max _{-1 \leq x \leq 1}\left|f(x)-f_{J-1}(x)\right| \leq \sum_{k=J}^{\infty}\left|a_{k}\right| \leq \frac{I_{2}(f)}{J^{2}}+\frac{I_{4}(f)}{J^{3}} .
$$

Several comments are now in order:

a) The functional (4.7) gives different weight to the boundaries than (3.5) and weights the second derivative differently from the first derivative.

b) The same analysis, though integrating by parts only once, shows that

$$
\left|a_{J}\right|^{2} \leq \frac{k}{J^{2}} \int_{-1}^{1} \frac{(L f)^{2}}{\sqrt{1-x^{2}}} d x
$$

where $\mathrm{K}$ is some constant. Thus even the lowest order.error bounded does not reduce to the weighted Sobolev norm. This may explain the lack of success of using the weighted Sobolev norm of the first derivative in $[1,6]$.

c) The bound (4.7) can be extended to bound the spectral interpolation error based on collocation, with the loss of at most a factor of $10 \mathrm{~J}$. This is because the right hand side of $(4.8)$ is also an upper bound for the best polynomial approximation to $f$ in the sup norm. It is known [10] that the maximum norm of the error of the interpolant of $f$, at the points (3.3) will differ from the error obtained from the minimax polynomlal, by a factor that grows at most logarithmically.

d) The analysis presented here is only for the spectral interpolation error. However, the numerical results indicate that minimizing (4.7) is effective in controlling the total error in our computations. 


\section{Numerical Pesults}

In this section we present some numerical results illustrating the effectiveness of (4.7) in tracking the spectral interpolation error. We conslder the model described by (2.1) and the parameters described in Section 2. In our adaptive procedure we consider the current coordinate system described by $\mathfrak{\alpha}=\mathfrak{Q}^{\prime}$. The solution is updated in time and $I_{2}(u)$ is computed at every time step. A search for a new coordinate system is triggered whenever

$$
\frac{I_{2}(u)}{I_{\text {min }}}<c_{1},
$$

or

$$
\frac{I_{2}(u)}{I_{\min }}>c_{2} \text {, }
$$

where $I_{\mathrm{min}}$ is the value of $I_{2}(u)$ at the previous time that a coordinate system was found. We observe that (5.1a) may indicate that the number of collocation points should be reduced in order to more efficiently compute the solution, whereas ( $5.1 \mathrm{~b}$ ) may indicate that more collocation points are required to achieve the desired accuracy. Both (5.1a) and (5.1b) indicate that the solution has changed sufficiently from the previous time a coordinate system was found, so that a new minimization should be attempted. For the computations used below, we took $c_{1}=.3, c_{2}=1.7$. At present only one coordinate system is used for both variables. The function actually used in the evaluation of $I_{2}(u)$ is a linear combination of $\odot$ and $C$. For the computations of pulsating flames presented below, we use $u=.3 \theta+.7 \mathrm{C}$. In addition, we specify a maximum number of collocation points, $J_{\max }$, which is to be used in case the program cannot meet the specified error.

In Figures $3 a$ and $3 b$, we display the temporal behavior of $\theta$ and $c$ for the same computation as shown in Figure 1. In these figures, we also plot the number of collocation points as $t$ varies. In Figure $3 a$, the choice of $\underset{\sim}{\alpha}$ is obtained from (4.7), while in Figure $3 b$ the choice of $\alpha$ was obtained from (3.5). The value of $\varepsilon$ in each case was 0.0025 .

It is apparent that the use of (4.7) is more sensitive to the behavior of the solution than the use of (3.5), and permits a more rapid reduction in the number of collocation points near the spike, where the maximum cost of the computation occurs. In fact, the computation for Figure $3 a$ required roughly $30 \%$ of the time of the computation for FIgure $3 \mathrm{~b}$.

In Figures $4 \mathrm{a}$ and $4 \mathrm{~b}$, we plot the spatial profiles for $\theta$ and $\mathrm{C}$ at the initial spike $(t \sim 2.9)$. In each case we took the spatial profile at the 
time that the functional was largest. The value of the square of the functional is indicted in the figures. The major source of spatial errors is the oscillations in the profiles, primarily in the concentration profile. We observe that the solution in Figure 4b (using (3.5)) is slightly more oscillatory than the solution in Figure $4 a$ (using (4.7)), although both solutions are acceptable. The effect of increasing the error tolerance is shown in Figures $5 a$ (using (4.7)) and 5b (using (3.5)). In this case we set $\varepsilon=.0035$. It can be seen that Figure $5 a$ is more accurate. In this case the maximum number of collocation points for Figure 5a was 321 while in Figure 5 b the error level was not met with 361 points (the maximum number of points allowed in this run of the program). The ratio of the runing time was 5 to 1 .

As another example, we reduced the maximum allowed number of collocation points to 241. Spatial profiles using (4.7) (Figure 6a) and (3.5) (Figure 6b) are illustrated. Although both solutions are degraded, it is apparent that Figure $6 \mathrm{a}$ is considerably more accurate than Figure $6 \mathrm{~b}$.

We next discuss the application of the method described above to the computation of stationary cellular flames. We consider (2.1), allowing both radial and angular perturbations, for values of $L<1$. We consider $L<L_{C_{1}}$ so that the axisymmetric solution to (2.1) is unstable to angular perturbations and arbitrary initial data sufficiently close to the axisymmetric solution, will evolve to a stable stationary cellular solution. These cellular flames, sometimes called wrinkled flames, are characterized by the appearance of pointed crests and troughs in the reaction zone. The crests point in the direction of the burned products of combustion and are cooler than the troughs. This behavior, described analytically by the interaction between the fundamental and its first harmonic [8], is also observed experimentally [7] and is present in our computations at finite activation energy as well $[2,3]$.

In our computations, we employ a Fourier pseudo-spectral method in the angular direction $\phi$ and a Chebyshev pseudo-spectral method in the radial direction $r$. At present we employ a transformation in the radial direction with $\underset{\sim}{\alpha}$ independent of $\phi$, and therefore we require sufficient radial resolution to resolve the reaction zone in each angular direction. The stationary state is achieved by integrating in time until the solution equilibrates. The convergence is monitored by the maximum of $\left|\frac{\partial \theta}{\partial t}\right|,\left|\frac{\partial C}{\partial t}\right|$, over all collocation points. We require this maximum to be less than $10^{-7}$. The temporal integration uses the backward Euler scheme with operator splitting. The reaction terms are treated explicitly while all other terms are treated implicitly [2].

We illustrate our method with a computation of a three cell. The parameter values employed are $K=11.0, \mathrm{~L}=0.44, \mathrm{~N}=20, \quad$ and $\sigma=0.615$. We first describe the behavior of the solution. Figures $7 a, b$ illustrate the 
temperature and concentration, as a function of $\phi$, at two different radial positions. We observe that for $r=9.34$, the temperature exhibits a large, nearly sinusoldal oscillation with few visible higher frequencies. For $r=$ 10.15 the angular variation is reduced, but the temperature exhibits crests and troughs. This is due to the interaction of the fundamental and its first harmonic; the other harmonics are very small. This effect is illustrated in Figure $7 \mathrm{c}$ by plotting the mean, fundamental, and first harmonic as a function of $r$.

The data in Figure 7 were obtained by using 101 points in $r$ and 128 collocation points in $\phi$. This solution has been validated by further grid refinements, and for this number of radial collocation points, the solution does not depend upon the specific functional used to determine the coordinate system. To illustrate the effectiveness of (4.7), we consider a cruder approximation, using a reduced number of collocation points.

We compare the fine grid solution with computations using (4.7) and (3.5) with 49 collocation points. The objective function is obtained by averaging the functional over all angles. In Figure $8 \mathrm{a}$ we plot the mean value of the solution, comparing the fine grid solution with the coarse grid solution obtained by using (4.7). In FIgure $8 \mathrm{~b}$ we do the same for the fundamental and its first harmonic. In Figures $9 a$ and $9 b$ we present the same data, except that the coarse grid solution is obtained by using (3.5).

It is apparent from the figures that the coarse grid solutions are considerably less accurate on the angular variations than on the mean. Although the first harmonic is small relative to the mean and the fundamental, we consider it an important part of the computation, as the pointed crests and troughs in the reaction zone are features predicted by analysis and by our computations and are observed experimentally. It is apparent from the figures that by using (4.7), we obtain a more accurate solution than by using (3.5). A more detailed numerical study of cellular flames is presented in $[2,3]$.

\section{Conclusion}

We have presented a functional that appears to provide a relatively sharp upper bound for the spectral interpolation error, in the maximum norm, for the types of solutions occurring in typical combustion problems. The functional is derived directly from the Chebyshev expansion of a smooth function; it is the first member of a family of functionals, depending upon successively higher derivatives, which bound the spectral interpolation error. We have employed the functional to both reduce the error for a fixed number of collocation points and to dynamically vary the number of collocation points, depending on the magnitude of the functional. The adaptive procedure based on 
this functional is shown to be more effective than the procedure based on the weighted Sobolev norm, in computations of both pulsating and cellular flames.

\section{Acknowledgement}

We thank Eli Turkel for critically reading the manuscript and for stimulating discussions on this topic.

\section{Beferences}

1. A. Bayliss and B. J. Matkowsky, J. Comput. Phys., Vo1. 71, p. 147 (1987).

2. A. Bayliss, B. J. Matkowsky, and M. Minkoff, Applied Math. Letters, to appear.

3. A. Bayliss, B. J. Matkowsky, and M. Minkoff, "Bistable cellular flames," in Symposium in honor of C. C. Lin, Proceedings of a Symposium, D. Benney, F. Shu, and C. Yuan, ed., World Scientific Pub., Philadelphia, 1987.

4. C. Canuto and A. Quarteroni, Math Comput., Vol. 38, p. $67 \cdot(1982)$.

5. D. Gottlieb and S. A. Orszag, CBMS-NSF Regional Conference Series in Applied Mathematics, No. 26, SIAM, Philadelphia, 1977.

6. G. H. Guillard and R. Peyret, Report No. 108, Universite de Nice, 1986.

7. G. H. Markstein (ed.), "Nonsteady flame propagation," Pergamon Press, NY, 1964 .

8. B. J. Matkowsky, L. J. Putnick, and G. I. Sivashinsky, SIAM J. Appl. Math., Vol. 38, p. 489 (1980).

9. B. J. Matkowsky and G. I. Sivashinsky, SIAM J. Appl. Math., Vol. 37, p. 686 (1979).

10. J. H. McCabe and G. M. Phillips, BIT, Vol. 13, p. 434 (1973).

11. M. D. Smooke and M. L. Koszykowski, SIAM J. Sci. Stat. Comput., Vol. 7, p. 301 (1986).

12. A. Solomonoff and E. Turke1, NASA CR 178179, ICASE Report 86-60, 1986.

13. E. Tadmor, SIAM J. Numer. Ana1., Vo1. 23, p. 1 (1986). 


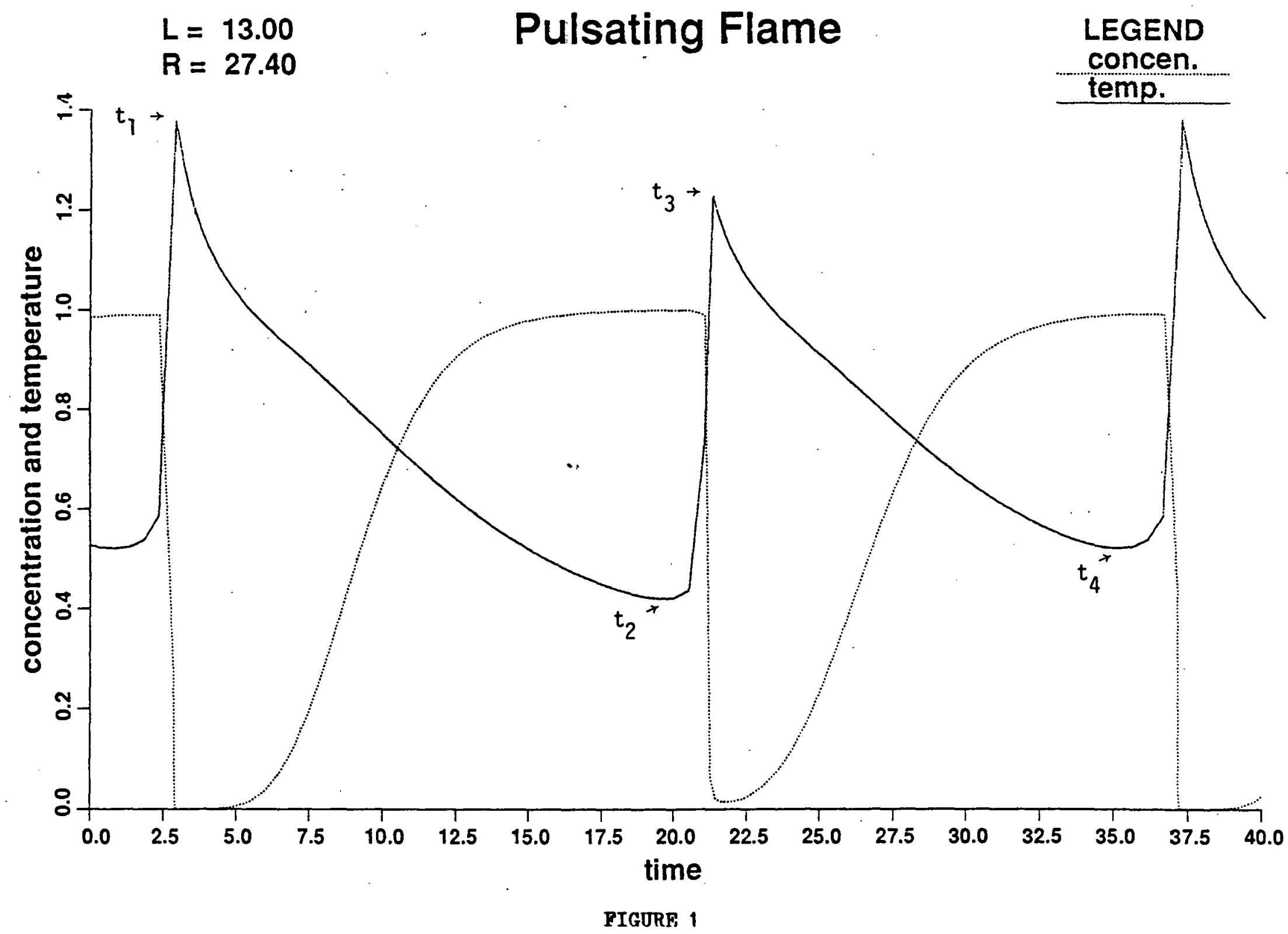




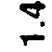

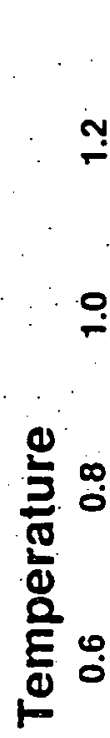

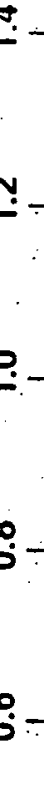

.

.

ֻั

$\stackrel{0}{0}$

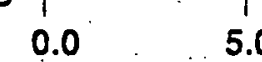

5.0
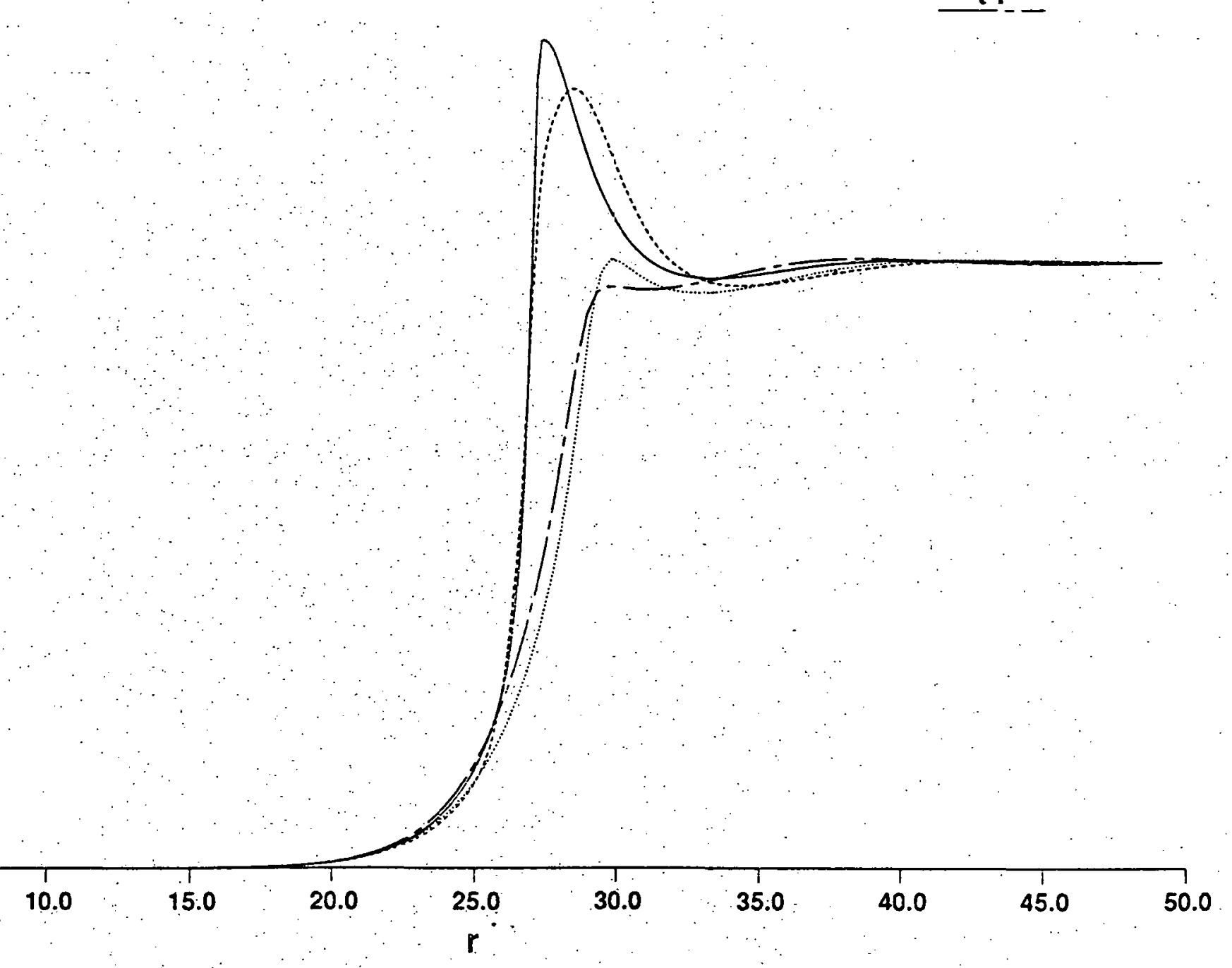

PIGURE 2a 


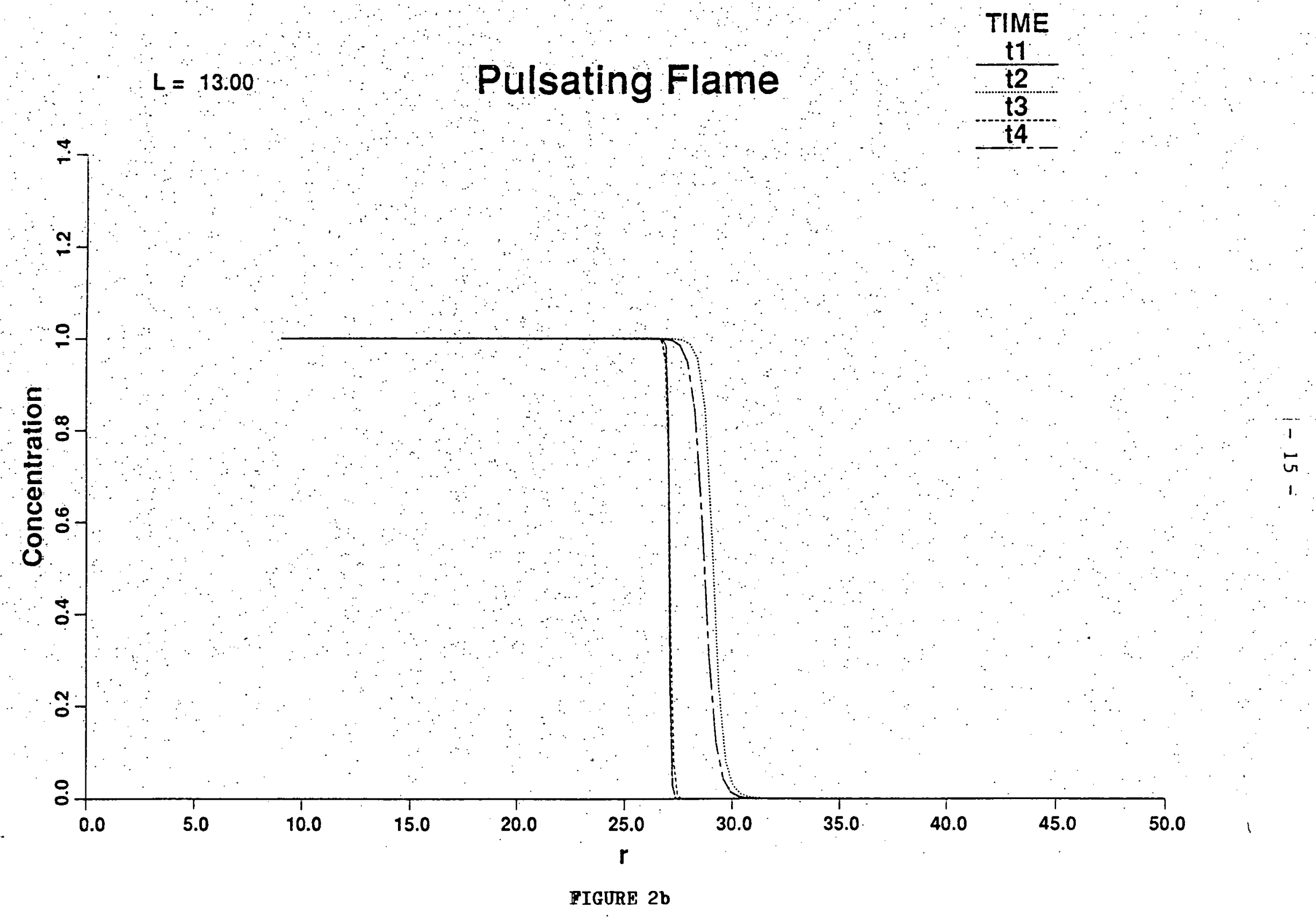




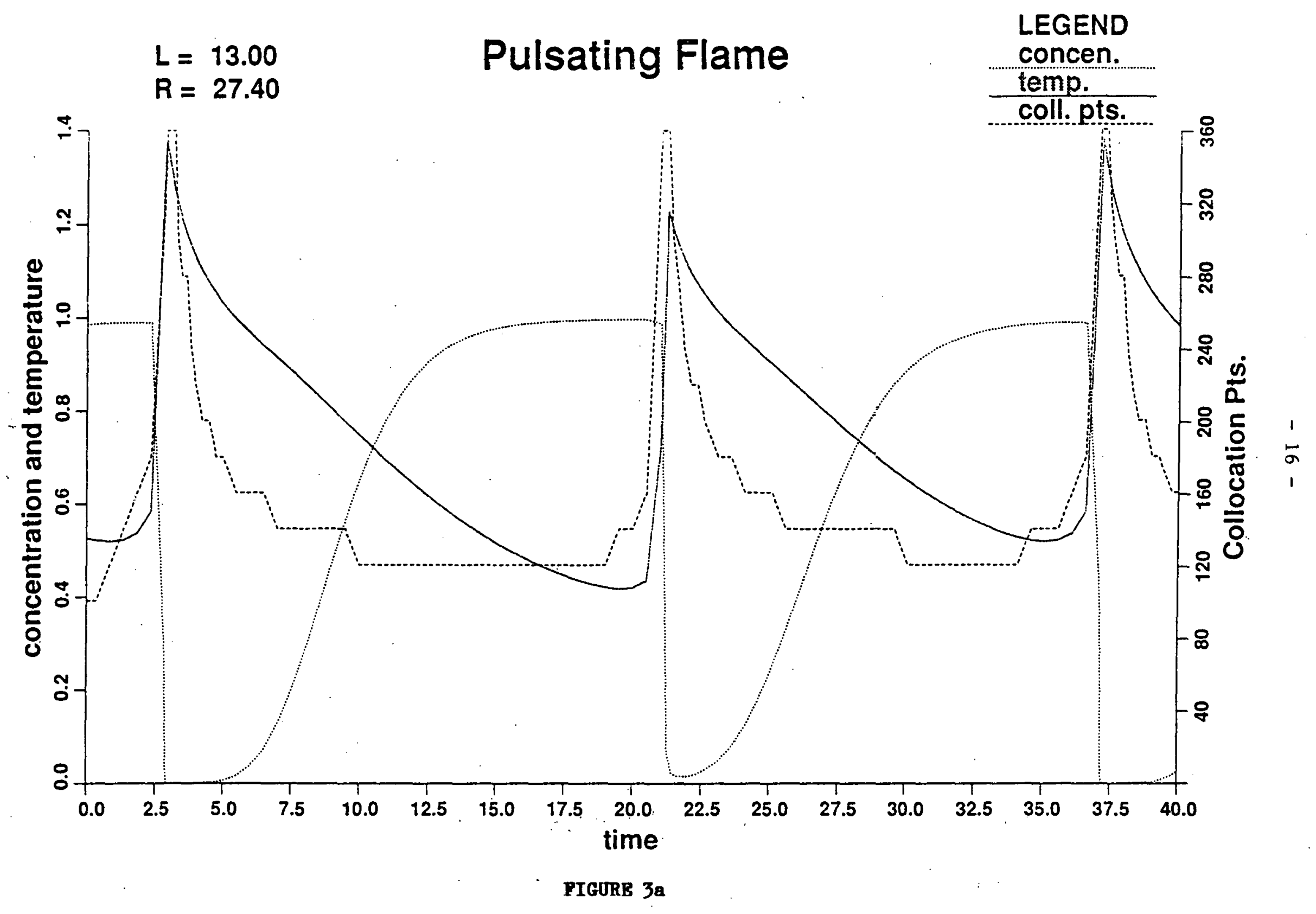




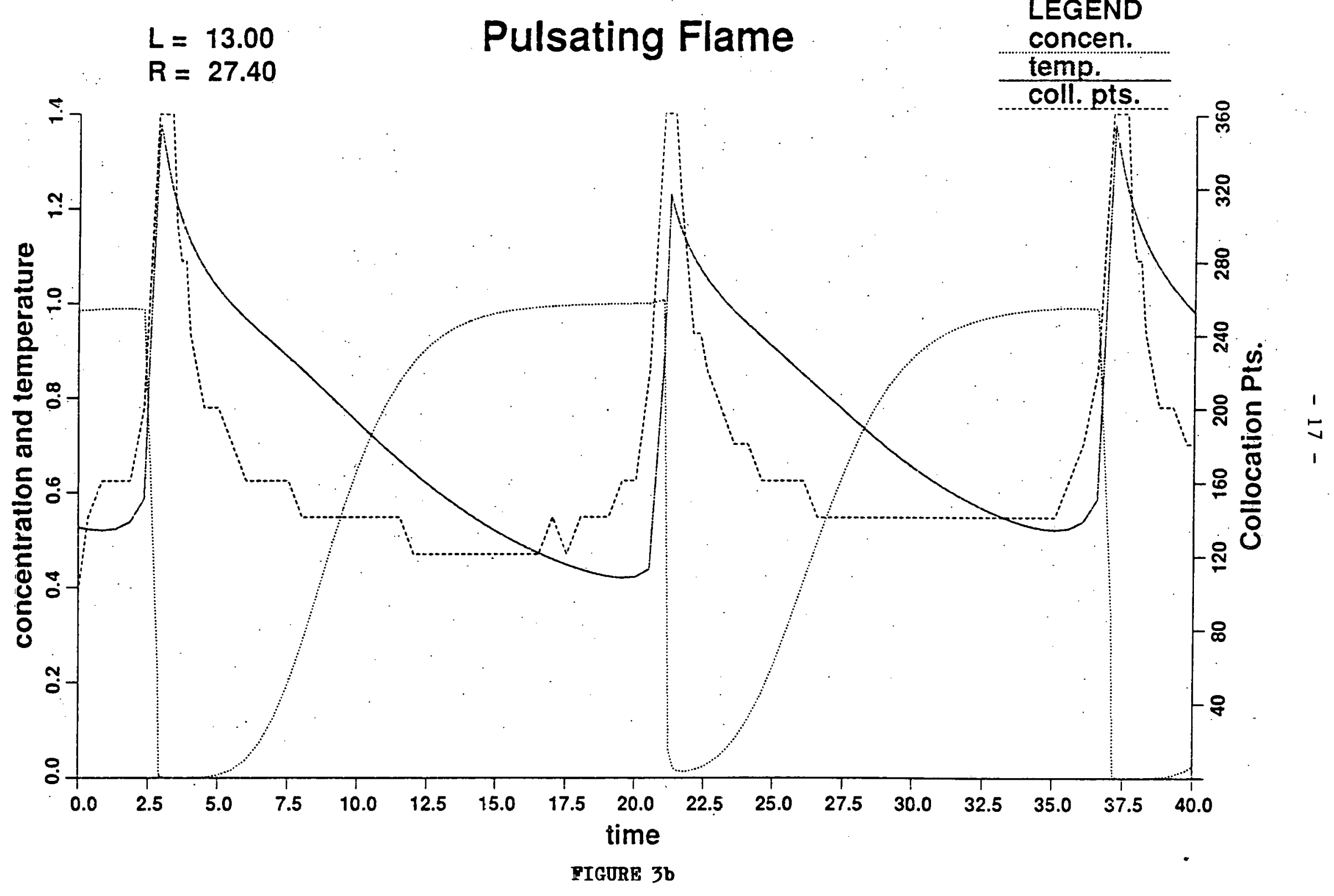




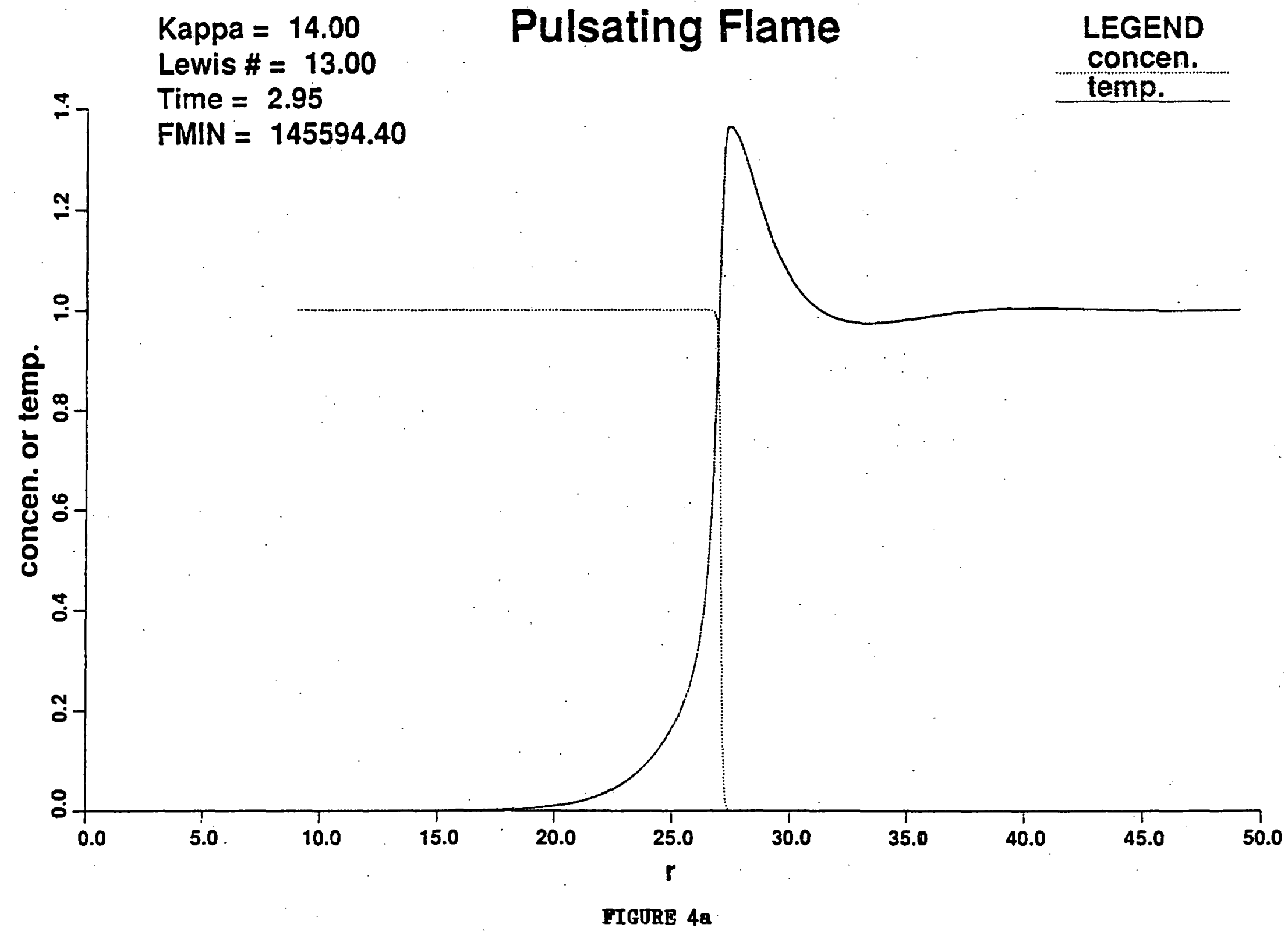




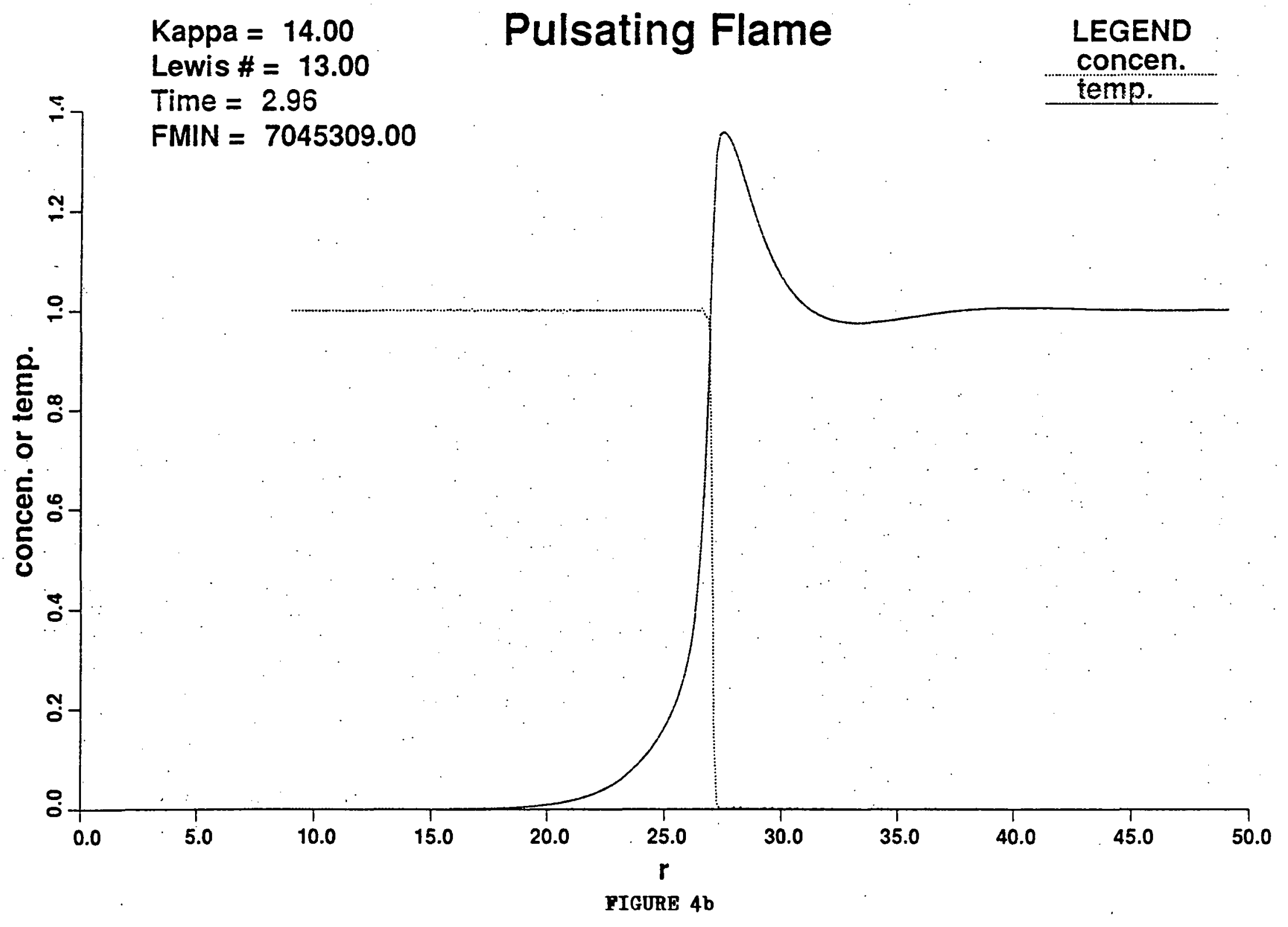




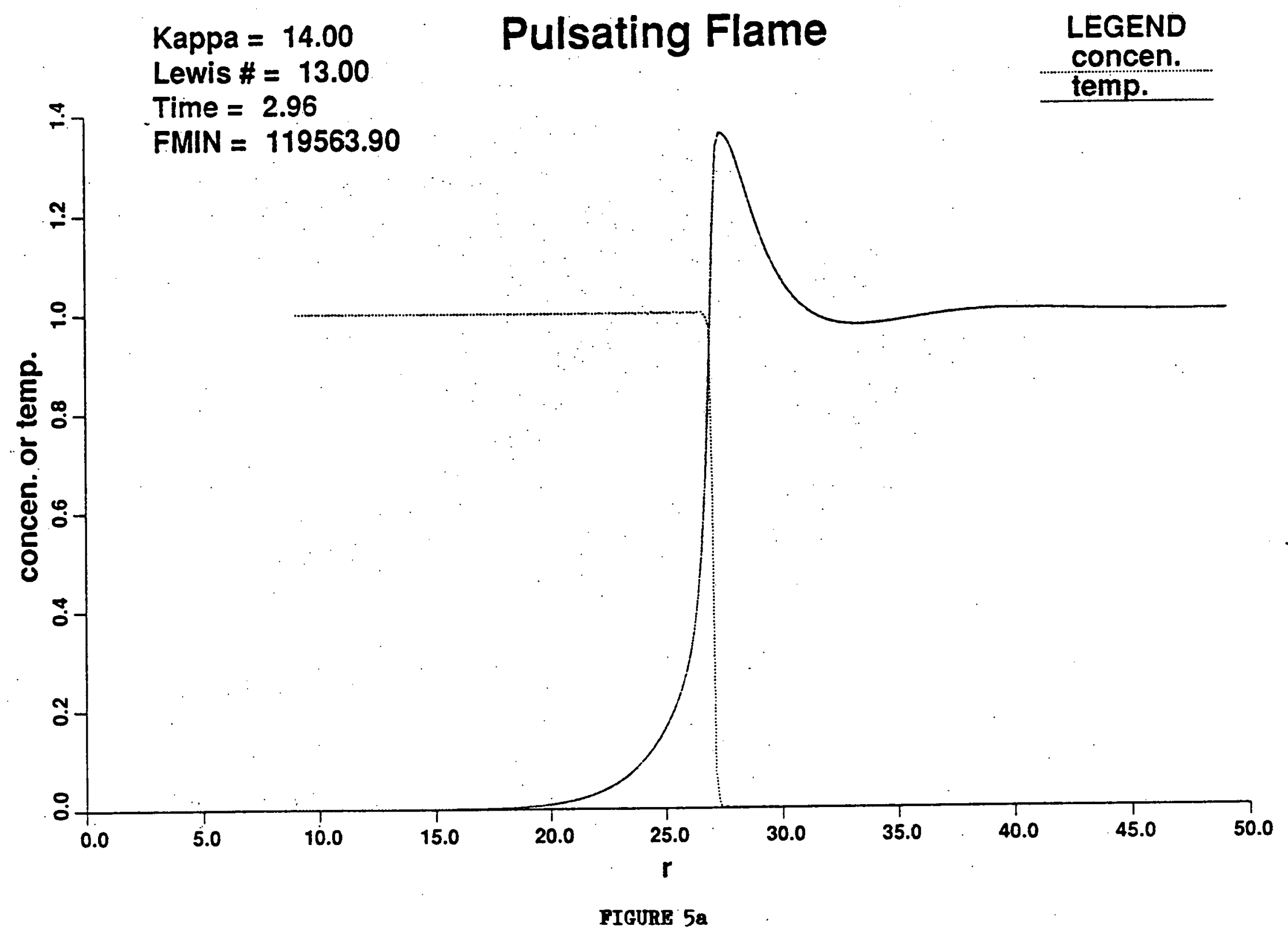




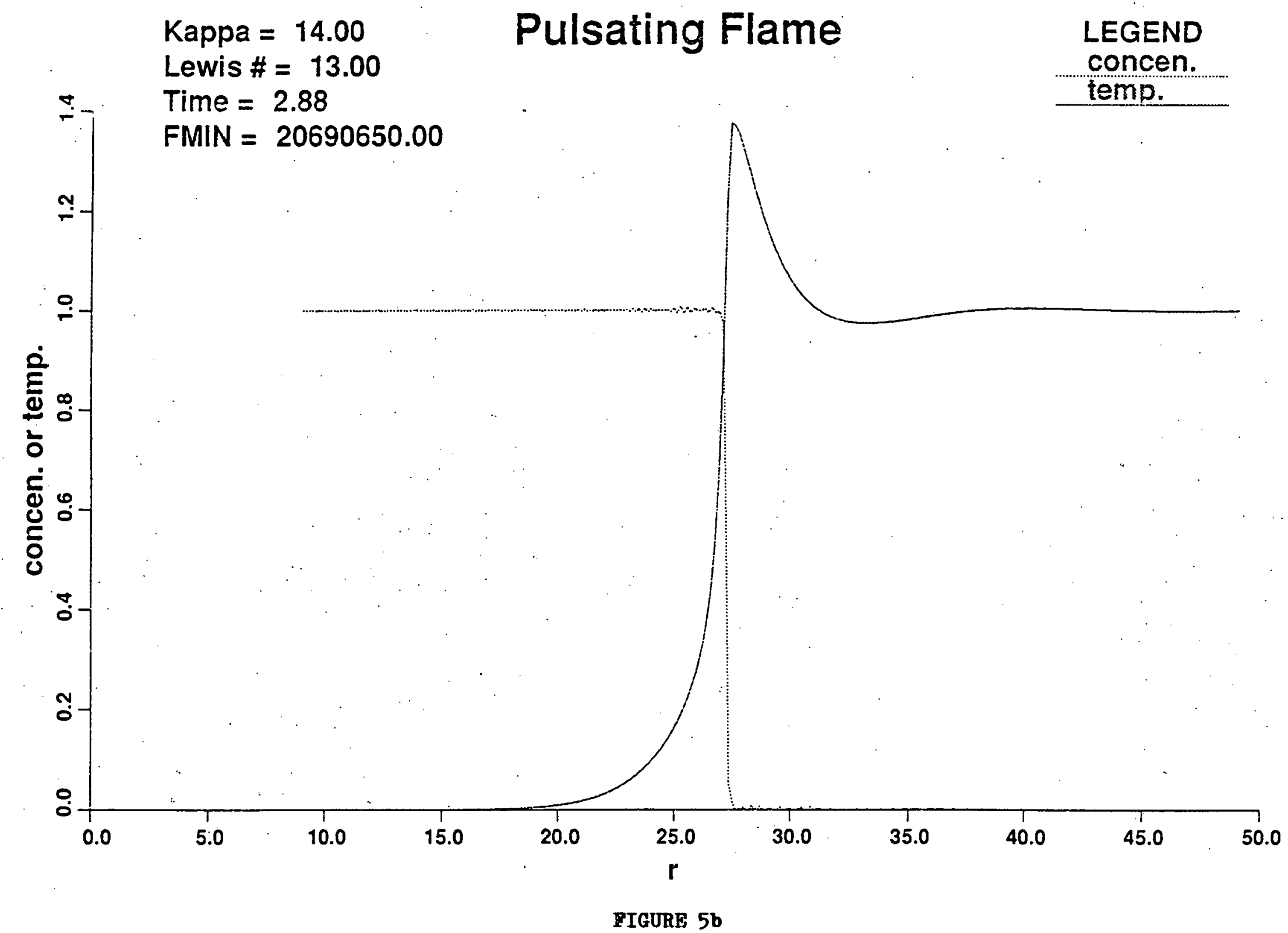




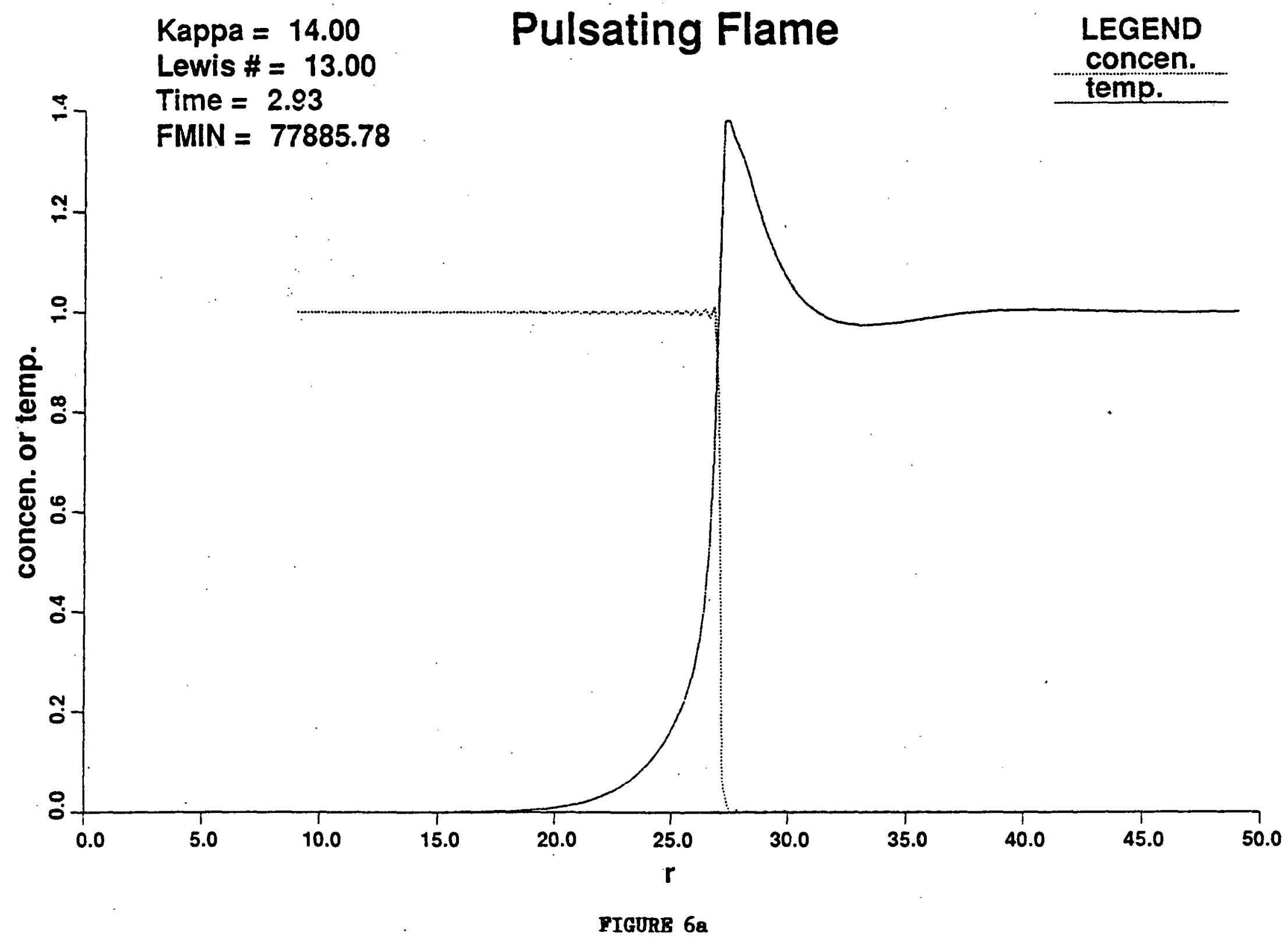




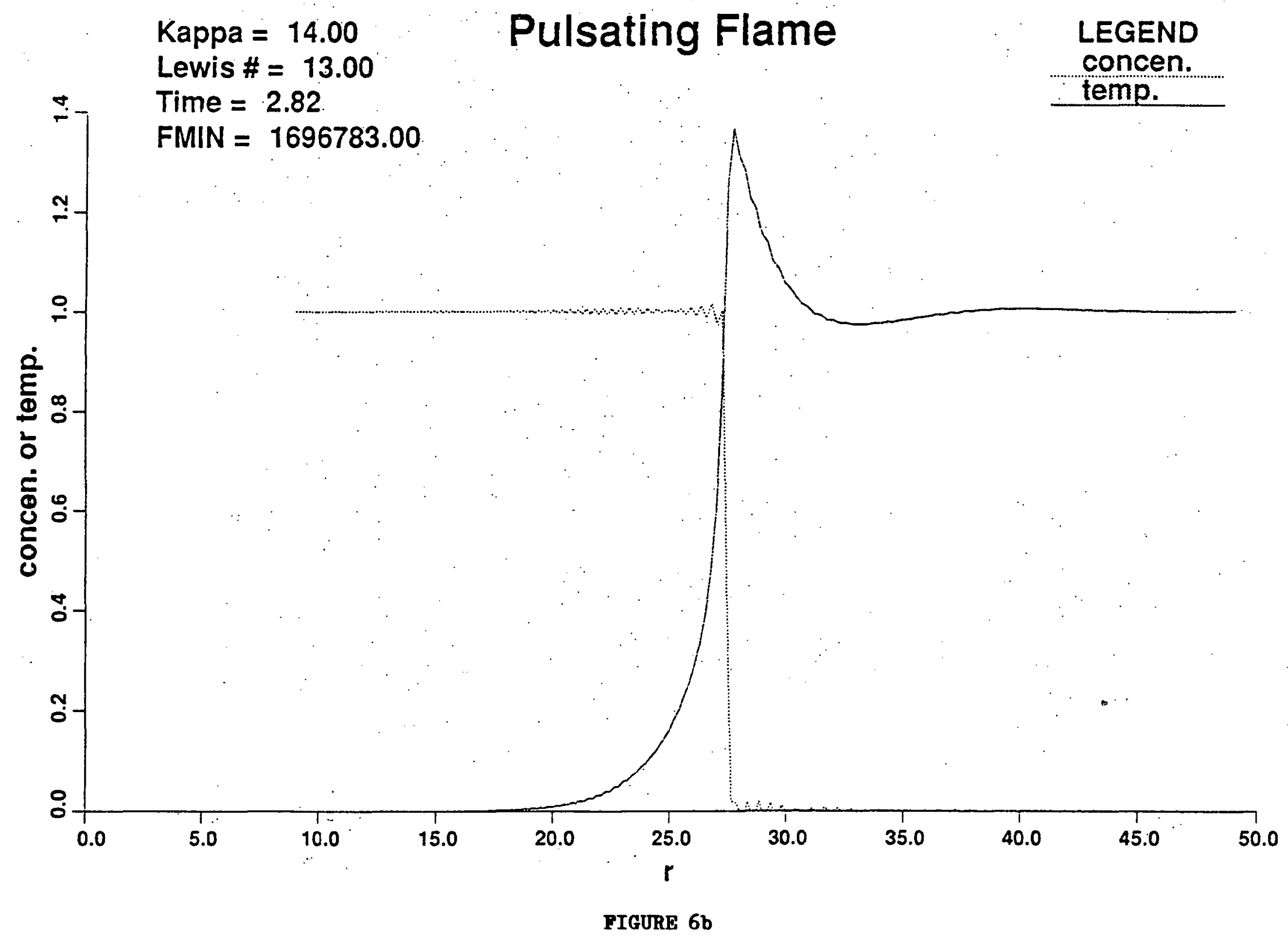




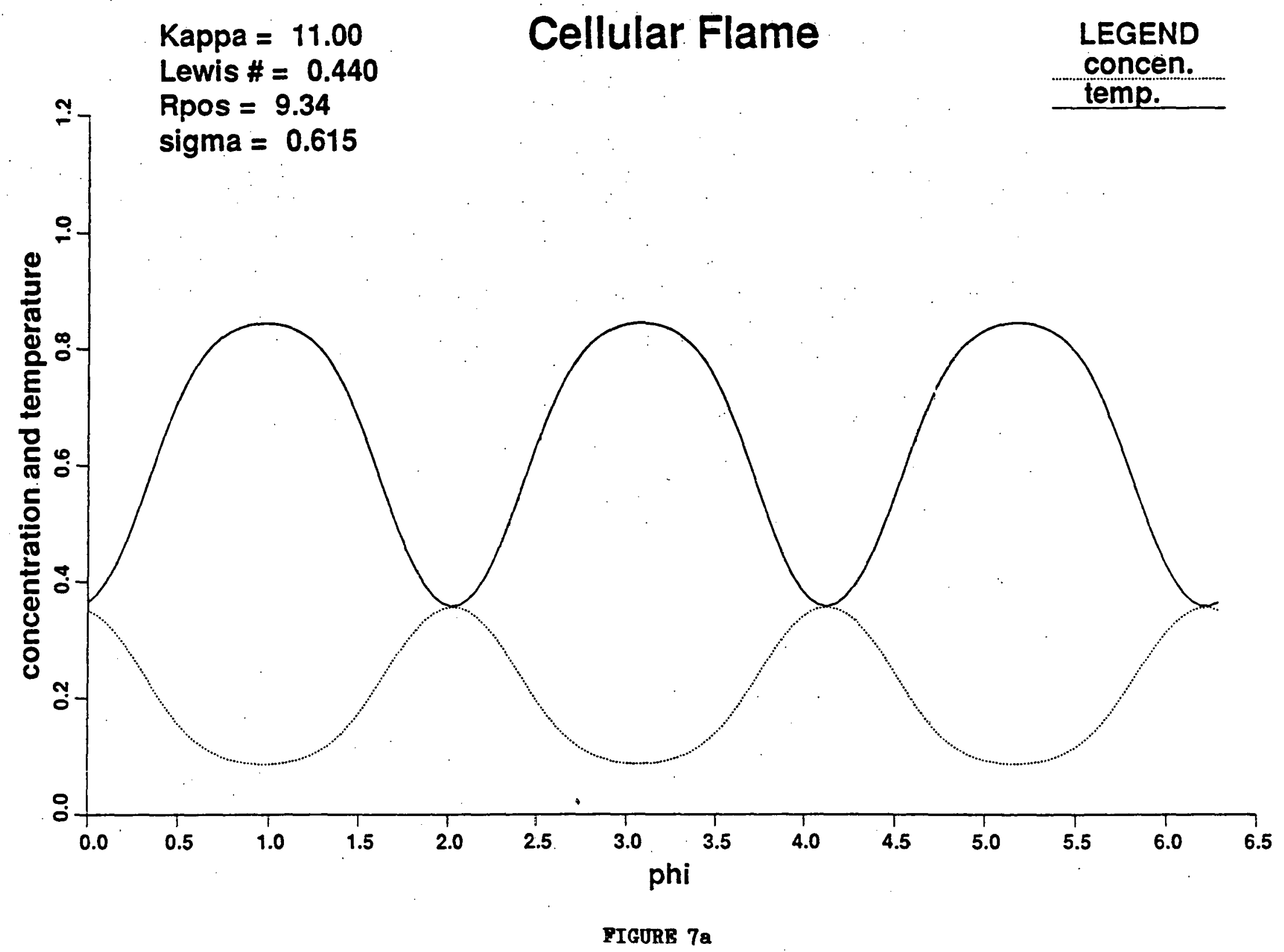




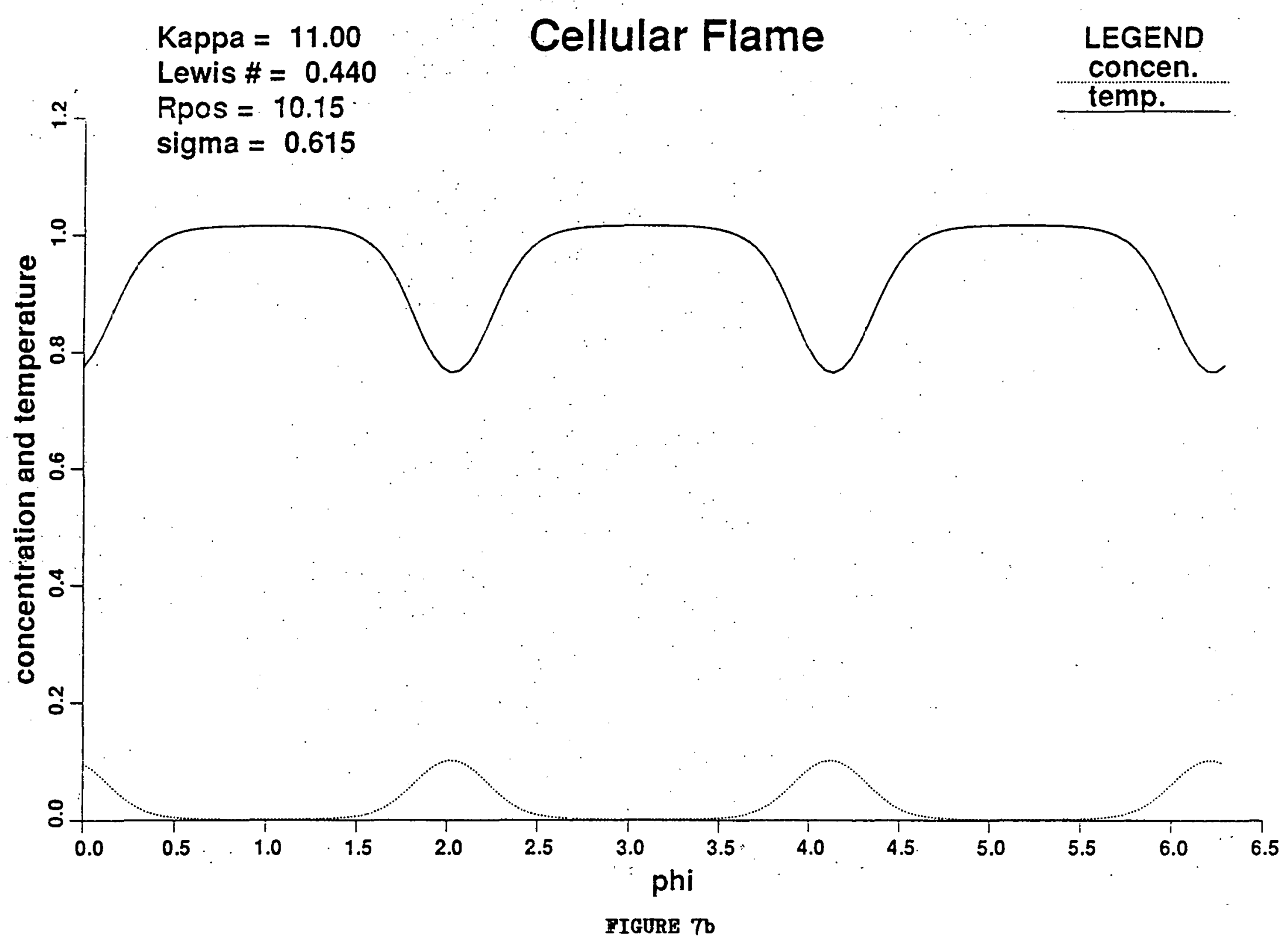




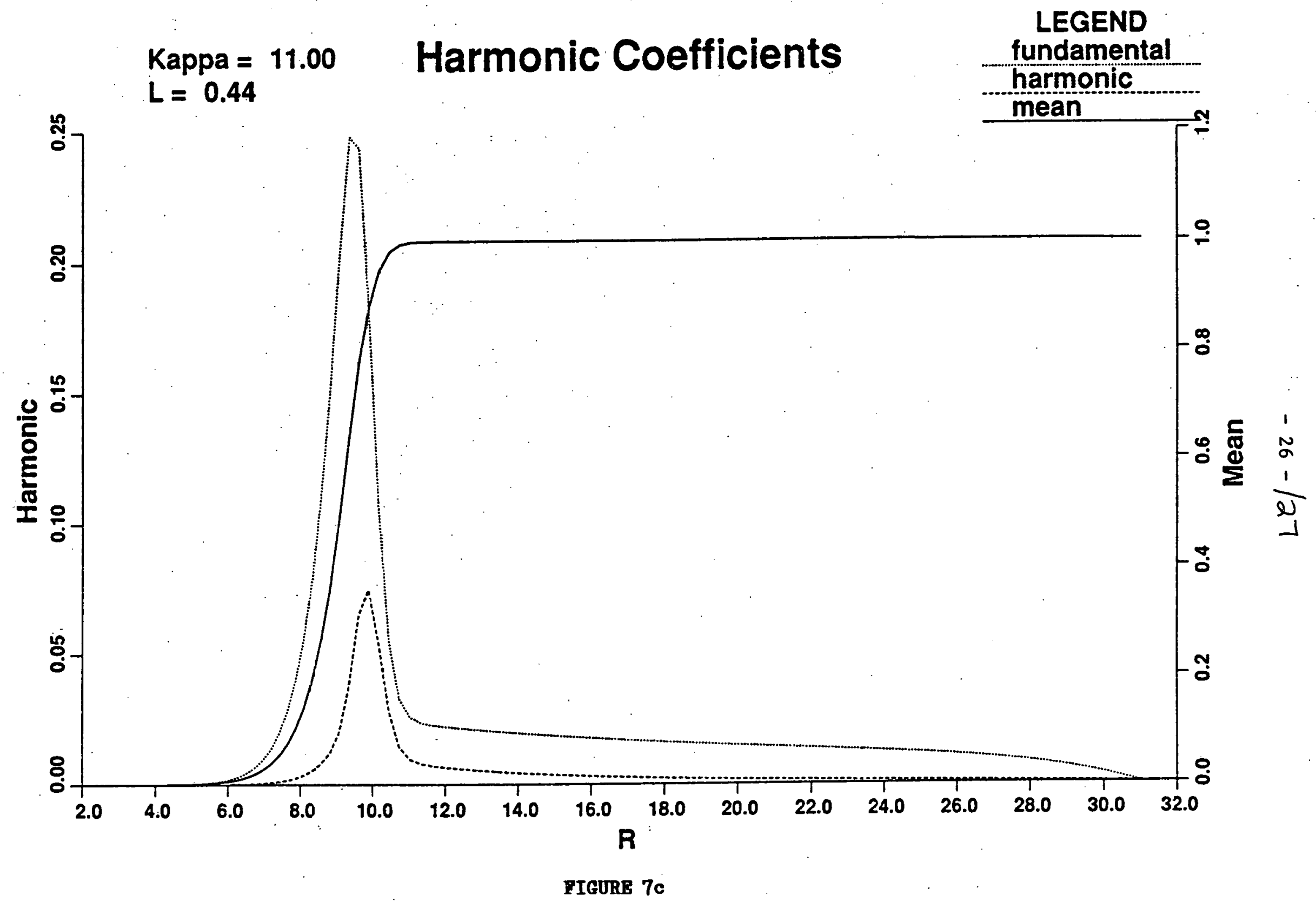




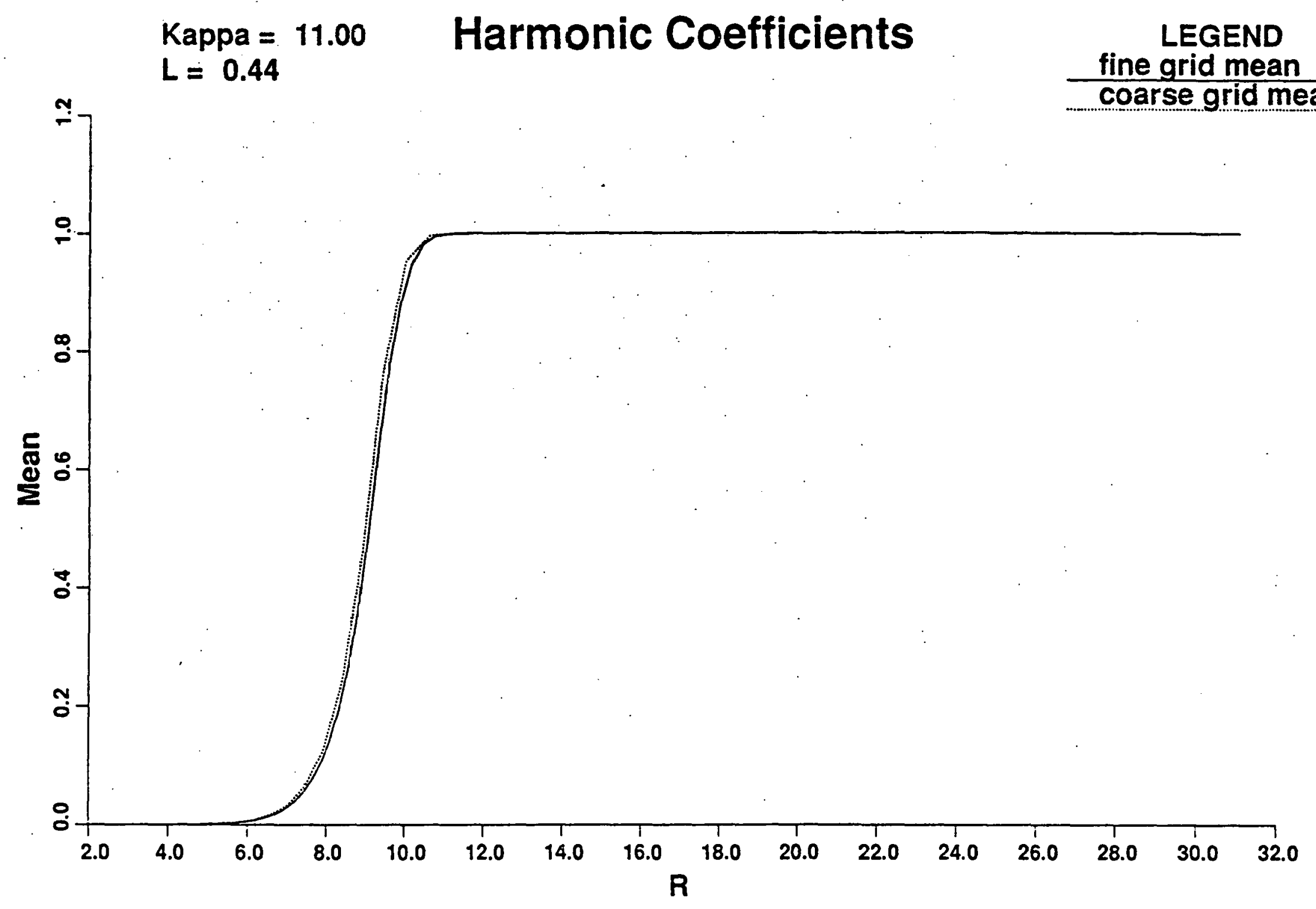

PIGURe 8a 
LEGEND

fine fundamental fine harmonic coarse fundament coarse harmonic

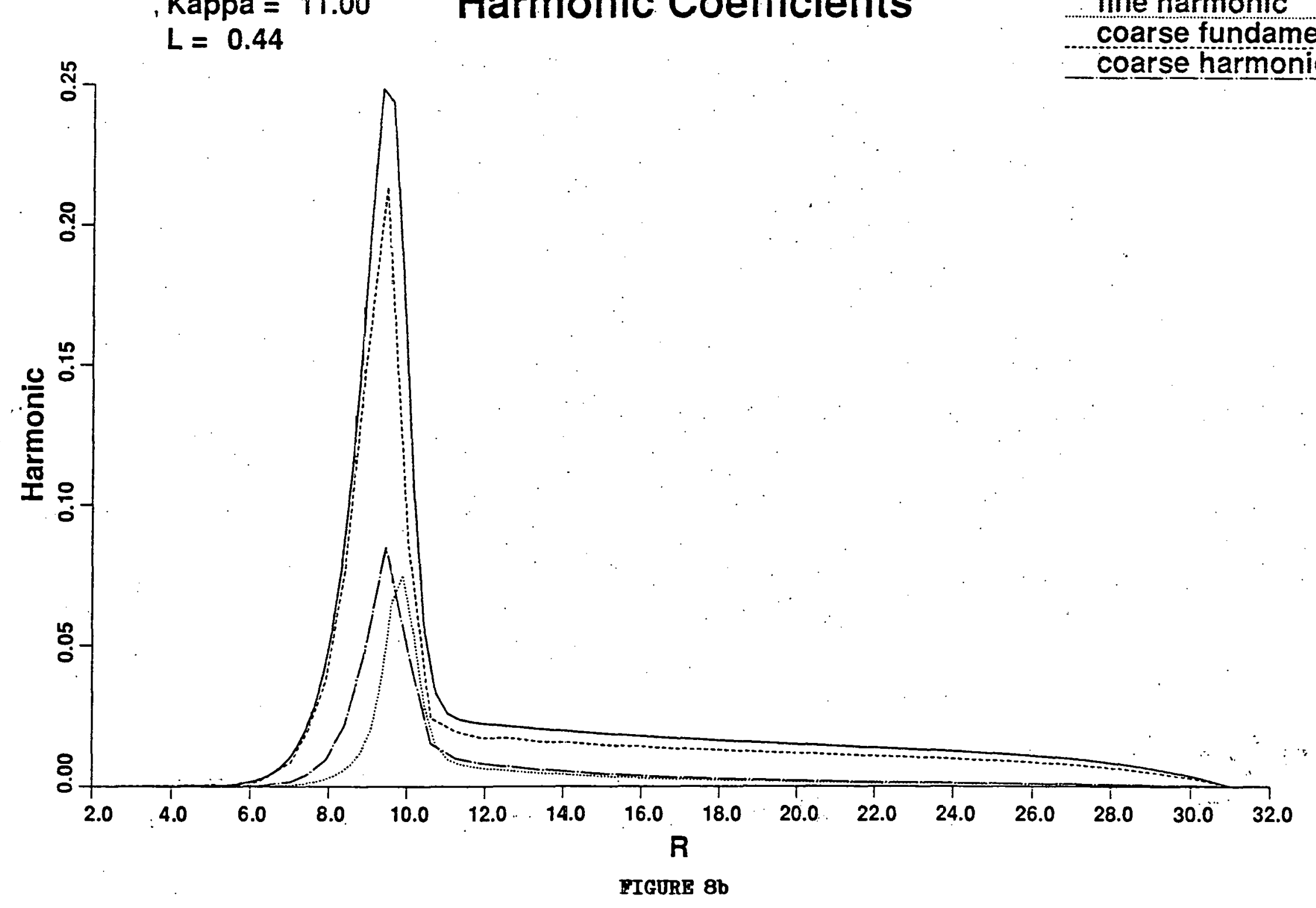




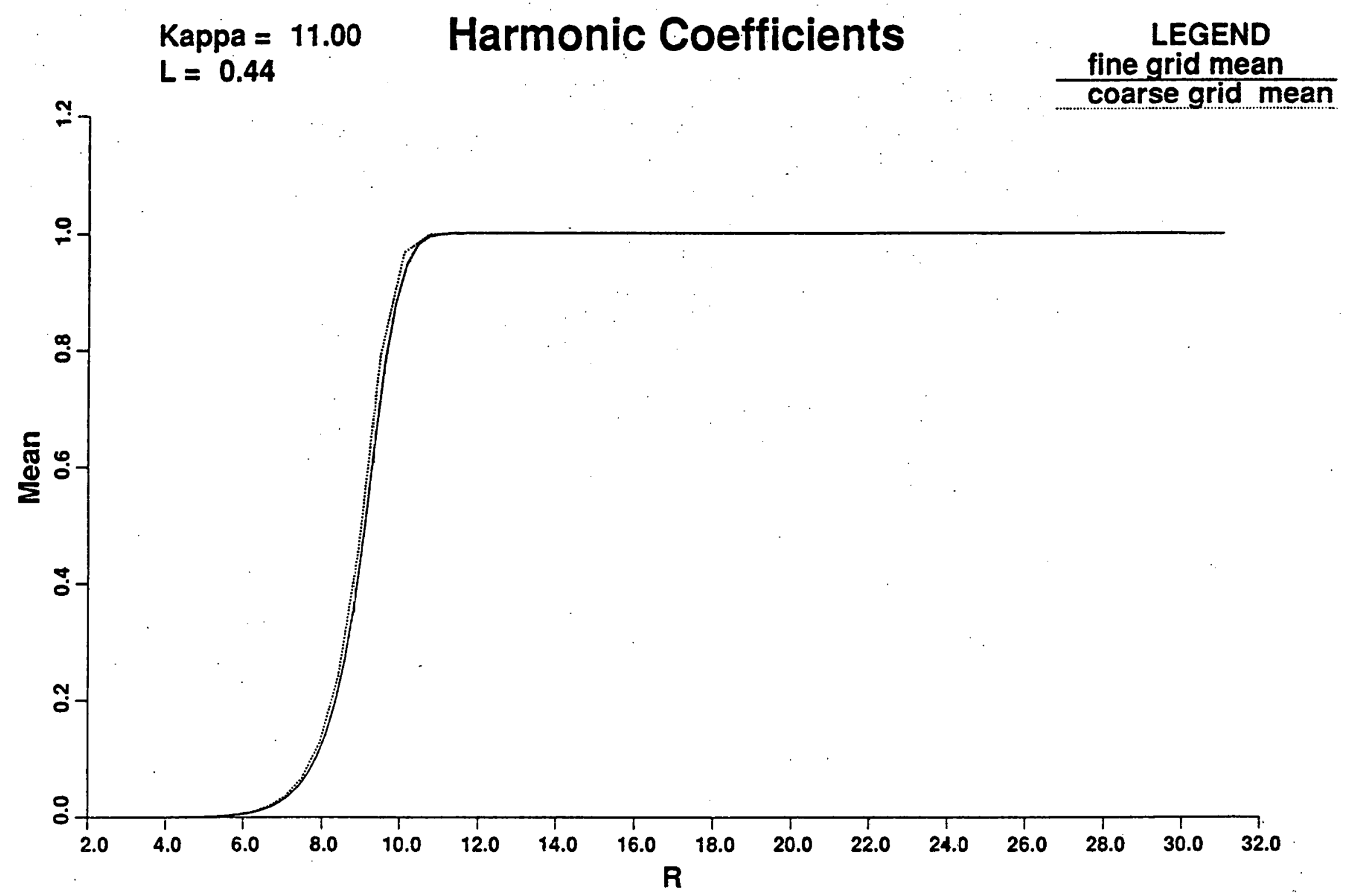


LEGEND

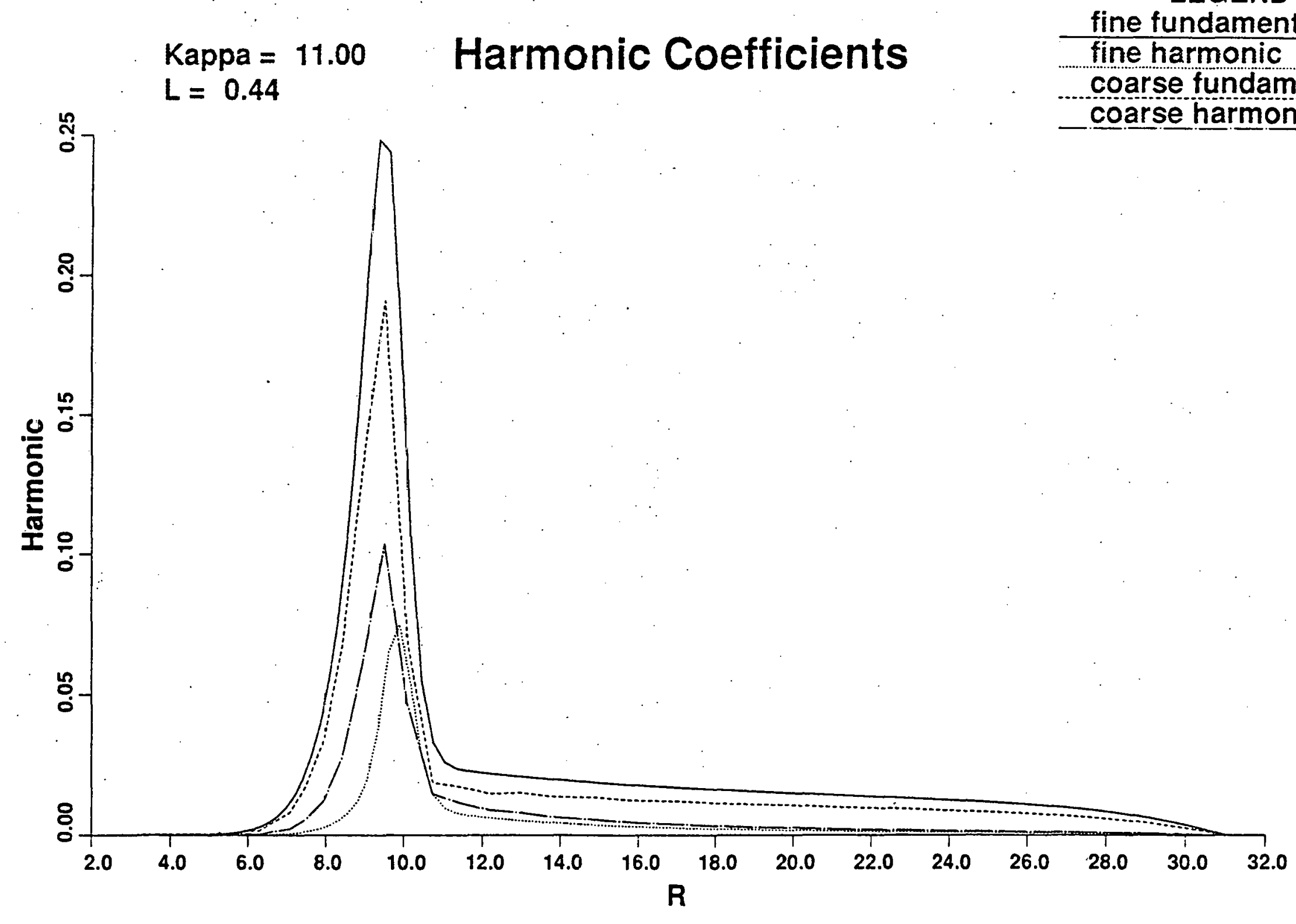

PIGURE 9b 